\title{
Alzheimer's disease biomarker discovery using in silico literature mining and clinical validation
}

\author{
Ines Greco ${ }^{1}$, Nicola Day², Joanna Riddoch-Contreras', Jane Reed², Hilkka Soininen³ , Iwona Kłoszewska ${ }^{4}$, \\ Magda Tsolaki ${ }^{5}$, Bruno Vellas ${ }^{6}$, Christian Spenger ${ }^{7}$, Patrizia Mecocci ${ }^{8}$, Lars-Olof Wahlund ${ }^{9}$, Andrew Simmons ${ }^{1}$, \\ Julie Barnes ${ }^{2,10}$ and Simon Lovestone ${ }^{1 *}$
}

\begin{abstract}
Background: Alzheimer's Disease (AD) is the most widespread form of dementia in the elderly but despite progress made in recent years towards a mechanistic understanding, there is still an urgent need for disease modification therapy and for early diagnostic tests. Substantial international efforts are being made to discover and validate biomarkers for AD using candidate analytes and various data-driven 'omics' approaches. Cerebrospinal fluid is in many ways the tissue of choice for biomarkers of brain disease but is limited by patient and clinician acceptability, and increasing attention is being paid to the search for blood-based biomarkers. The aim of this study was to use a novel in silico approach to discover a set of candidate biomarkers for AD.

Methods: We used an in silico literature mining approach to identify potential biomarkers by creating a summarized set of assertional metadata derived from relevant legacy information. We then assessed the validity of this approach using direct assays of the identified biomarkers in plasma by immunodetection methods.

Results: Using this in silico approach, we identified 25 biomarker candidates, at least three of which have subsequently been reported to be altered in blood or CSF from AD patients. Two further candidate biomarkers, indicated from the in silico approach, were choline acetyltransferase and urokinase-type plasminogen activator receptor. Using immunodetection, we showed that, in a large sample set, these markers are either altered in disease or correlate with MRI markers of atrophy.
\end{abstract}

Conclusions: These data support as a proof of concept the use of data mining and in silico analyses to derive valid biomarker candidates for $A D$ and, by extension, for other disorders.

Keywords: Alzheimer's disease, Proteomics, Biomarkers, Choline acetyltransferase (ChAt), Urokinase-type plasminogen activator receptor (PLAUR), Intelligence network, Bioinformatics, MRI, in silico, Literature mining

\section{Background}

Alzheimer's disease (AD) is one of the commonest causes of dementia resulting in a severe loss of intellectual abilities including memory. The main histological features of $\mathrm{AD}$ in brain are amyloid plaques and neurofibrillary tangles, due to accumulation, respectively, of amyloid beta $(A \beta)$ peptide and tau protein in insoluble form. The causes of this, and other pathology found in the $\mathrm{AD}$ brain, are not known with certainty but are likely to be multifactorial. This multifactorial and only partially

\footnotetext{
* Correspondence: simon.lovestone@kcl.ac.uk

'King's College London, Institute of Psychiatry, De Crespigny Park, London SE5 8AF, UK

Full list of author information is available at the end of the article
}

understood pathogenesis complicates both drug and biomarker discovery.

The search for biomarkers to aid accurate diagnosis, predict progression and for use in clinical trials has become a major research goal $[1,2]$. The most widely used strategy for the discovery of biomarkers is predicated on the identification of potential candidate biomarkers using knowledge of disease processes followed by validation, comparing healthy control to affected subjects [3]. In many respects, the optimal source of human tissue for the investigation of $\mathrm{AD}$ biomarkers is cerebrospinal fluid (CSF) and the demonstration of lowered CSF A $\beta$ and raised CSF tau and phosphorylated tau in $\mathrm{AD}$ is the prime example of candidate biomarker discovery and

\section{Biomed Central}


validation leading to qualification using an affected casenormal elderly control design [4].

However, all three approaches - candidate discovery, case-control design and the use of CSF have their drawbacks. CSF although ideal in many respects as it bathes the diseased organ, is limited by patient and clinician acceptability, especially for repeated measurements. Affected case versus normal elderly control experimental design is severely limited by the long prodromal phase in $\mathrm{AD}$, meaning many apparently unaffected controls may have substantial occult pathology. Candidate biomarkers are limited by the complex multifactorial pathology in $\mathrm{AD}$ meaning that beyond $\mathrm{A} \beta$ and tau it becomes difficult to ascertain true candidates. In an effort to overcome the limitations of CSF, we, and others, have pursued, with some early indications of success, markers in plasma that might act as biomarkers of disease [5]. To overcome the problems of occult pathology and to identify biomarkers of potential utility, two broad approaches have been employed; firstly comparing people with prodromal states, for example in mild cognitive impairment $(\mathrm{MCI})$ who progress to $\mathrm{AD}$, to those who do not, and secondly comparing both affected and asymptomatic people by a non-clinical marker of disease such as MRI measures of atrophy or PET measures of amyloid load [6,7]. This latter approach has been described variously as an endophenotype or extreme phenotype design. To mitigate the limitations of the candidate marker approach many have used a range of datadriven technologies including proteomic and genomic platforms and increasingly these datasets are being aggregated for biomarker discovery and understanding pathogenesis; for example in renal disease where over 200 datasets are mined in a web-based application to identify disease associated proteins [8].

Here we report a novel approach to biomarker discovery through the utilization of data-driven biomarker discovery in silico followed by validation in blood using automated analysis of structural MRI as an endophenotype measure of disease. The in silico study started by generating massive volumes of assertional data represented in the form of an Intelligence Network. Assertions are simple factual representations of statements made in the biomedical literature and other sources. They can be compiled into a semantically consistent form by applying comprehensive vocabularies and lexical matching approaches to yield a navigable database known as an Intelligence Network (IN). For this study, the IN contained assertions relating to proteins expressed in the brain and associated with pathology relevant to $\mathrm{AD}$. These are two important features for an ideal biomarker for $\mathrm{AD}$, assuming that such proteins could be measured in readily accessible fluids such as blood or urine. An in vitro study of two putative markers identified by the in silico screen - Choline Acetyltransferase (ChAt) and urokinase-type Plasminogen Activator Receptor (PLAUR) - provided evidence supporting the validity of the method suggesting that in silico screening for biomarkers in $\mathrm{AD}$ and, by extrapolation, other disorders, is a productive approach.

\section{Methods}

\section{In silico discovery of candidate biomarkers}

To identify a set of candidate biomarkers for AD, we applied an informatics approach that would enable a comprehensive analysis of a body of information embedded in publicly available literature sources and other information databases relevant to AD. The approach used resulted in the generation of thousands of highly accurate semantically consistent observational facts, known as assertions, which are represented in the form of subjectverb-object constructs and referenced back to the original source(s) e.g. "Amyloid Deposition_IS ASSOCIATED WITH_Alzheimer's Disease"; "BACE-1_IS INVOLVED IN_Amyloid Formation"; "Syntax in 1_IS EXPRESSED IN_Hippocampus".

Behind each assertion is a rich vocabulary that renders that assertion semantically consistent with the other assertions of the same nature. For example, the pathology term Amyloid Deposition can be described within the literature in a variety of ways as 'abnormal deposition of amyloid plaques', 'amyloid infiltration, 'amyloid deposits, 'amyloid protein deposition' etc. Amyloid Deposition is defined as the preferred 'concept', and all other terms link to that concept to generate a semantically consistent IN. All concepts are defined as a Concept Type e.g. in the case of Amyloid Deposition, a Pathological Observation. The result is a comprehensive and unbiased overview of relevant published data relating to $\mathrm{AD}$, expressed in a format that can be readily navigated, searched and analyzed.

\section{Scope of the intelligence network}

To define the scope of the IN relevant to the identification of candidate biomarkers of $\mathrm{AD}$, we set the criteria for the ideal biomarker. We defined that any biomarker for $\mathrm{AD}$ would ideally be a protein with a known role in the pathological development of the disease and have expression patterns within the brain that correlate with the localized hallmarks of AD pathology. We also defined an interest in proteins that had been reported to show patterns of upregulation. We made assumptions that such proteins could be ultimately identified in accessible fluids such as serum or urine. Thus, the IN that was generated linked data relating to protein/mRNA expression in relevant brain structures (e.g. hippocampus) with relevant pathological observations (e.g. loss of memory and tau phosphorylation). A network map of key 
Concept Types was then defined (See Figure 1) and this guided the build of the IN.

\section{Assertion generation}

The derivation of assertions from a body of historic information is challenging for a number of reasons. Firstly, the vocabulary used across the medical literature is highly varied and is typified by the use of synonyms, abbreviations, and idiosyncratic acronyms. Secondly, biomedical writing styles are diverse, with often complex linguistic patterns, involving descriptive terms, extended phrases, and statements or assertions that cross more than one sentence. There has been much effort invested in development of automated text mining methods that yield both sufficiently high accuracy and good recall. Our pragmatic approach is semi-automated i.e. automated extraction of possible subject-verb-object relationships (powered by rich vocabularies and synonym associations) is followed by manual validation.

To enable lexical matching and to deal with the synonym variations across the data sources of interest, a rich set of pre-curated vocabularies was developed, relating to subtypes of $\mathrm{AD}, \mathrm{AD}$-related pathological observations, brain regions affected by $\mathrm{AD}$, and proteins (and associated genes/mRNAs). Sample vocabularies used for Pathological Observations and Tissue are illustrated in Table 1. The vocabularies for Protein/mRNAs are too numerous to be listed, but comprised all known human proteins as defined in the publicly accessible protein database UniProt [9]. Each Concept was associated with a comprehensive set of synonymous terms.

In addition to the use of vocabularies around Proteins, Pathological Observations and Tissues, the assertiongeneration procedure also relied on the use of specific verbs or relationships (again with synonyms) between the key Concept Types. The preferred relationships for assertions stating a relationship between $\mathrm{AD}$ and various Pathological Observations included 'AFFECTS, 'CAUSES', 'HAS FEATURE, 'LEADS TO,' 'RESULTS IN'. For assertions between Anatomical Structure and Protein/mRNA, relationships such as EXPRESSES, HAS CONSTITUENT, IS LOCATION OF, IS SITE OF ALTERATION OF, HAS UPREGULATED were used. For assertions between Protein/mRNA and AD, relationships such as IS AFFECTED IN, IS EXPRESSED IN, IS ALTERED IN, IS COMPONENT OF, IS HIGHER IN, IS INCREASED IN, IS RISK FACTOR FOR, IS LOCATED IN were used.

To support the assertion generation process, a range of publicly accessible information sources were identified. These are detailed in Table 2 and included textual sources, such as full-text literature review papers, Medline abstracts, and reports from a variety of web-based $\mathrm{AD}$ research forums, as well as structured databases, such as gene expression databases (NCBI GEO), proteinpathway databases (e.g. GO, KEGG) and protein-disease association databases (e.g. OMIM). All sources provided both vocabularies and relevant assertions. The assertion generation process used the Sofia platform (see http:// www.biowisdom.com/tag/sofia/ and [10]) and was powered by the broad vocabularies around Proteins, Observations and Tissues, to enable semantic consistency of all final assertions. All the data sources were accessed between July 2006 and October 2006.

For unstructured data sources such as Medline, both lexical (pattern-matching) and linguistic (part-of-speech identification) techniques were used to extract relationships that exist between any of the Concepts in the network map. Several repeat extraction methods were applied, each using a different pattern of noun phrase, thus allowing for the diversity of language patterns used. The result was a high level of recall. The extraction procedure yielded a set of "proto-assertions", composed of

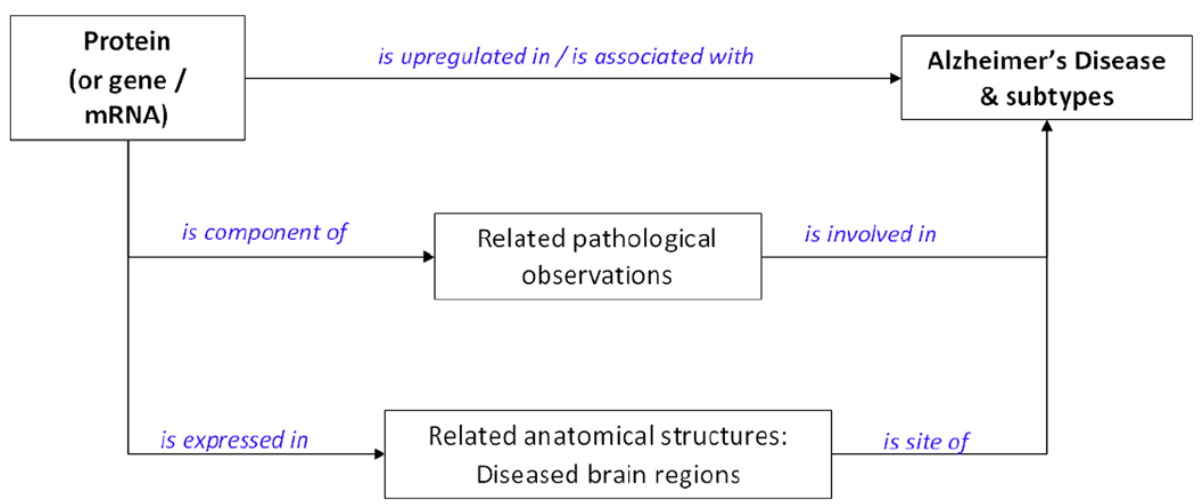

Figure 1 Network map used to guide the assertion generation for an Intelligence Network relevant for the discovery of candidate biomarkers for AD. Assertions could be direct links between proteins/mRNAs and AD (and sub-types), or indirect links via gene mutations (e.g. for familial forms of AD, such as OMIM record \#104300), via pathological processes (e.g. neuronal degeneration, amyloid formation, neurofibrillary tangles, hippocampal atrophy, tau deposition), or via anatomical structures (e.g. hippocampus, CA3 region, thalamus, temporal lobe). The relations (shown in blue) are examples of the vocabularies used in the assertions. 
Table 1 Sample vocabularies for key AD disease, pathological observations, and tissue concepts, used in the AD IN

Alzheimer's Disease sub-types

Alzheimer's Disease

Early Onset Alzheimer's Disease

Early Onset Familial Alzheimer's Disease

Familial Alzheimer's Disease

Incipient Alzheimer's Disease

Late Onset Alzheimer's Disease

Late Onset Sporadic Alzheimer's Disease

Mid-Stage Alzheimer's Disease

Mild-to-Moderate Alzheimer's Disease

Moderate Alzheimer's Disease

Moderate-to-Severe Alzheimer's Disease

Sporadic Alzheimer's Disease

Severe Alzheimer's Disease

\section{Pathological observations in AD}

Acute-Phase Reaction

Amyloid Deposition

Amyloid Fibril Formation

Amyloidosis

Asymmetric Cortical Atrophy

Blood Brain Barrier Dysfunction

Central Nervous System Inflammation

Cerebral Atrophy

Cholinergic Dysfunction

Corpus Callosum Atrophy

Dystrophic Neuronal Growth

Glial Inflammation

Gliosis

Glucose Hypometabolism

Granulovacuolar Degeneration

Hippocampal Neurodegeneration

Inflammation

Locus Coeruleus Neuronal Loss

Mitochondrial Failure

Nerve Degeneration

Neuritic Plaque Formation

Neurofibrillary Degeneration

Neurofibrillary Lesion

Neurofibrillary Tangle Formation

Neuroinflammation

Neuronal Degeneration

Neuronal Dysfunction

Neuronal Dystrophy

Neuronal Inclusion Bodies

Neuronal Lesion

Neuronal Loss

Neuronal Necrosis

Neuronal Shrinkage

Occipital Atrophy

Oxidative Damage

Oxidative Stress

Perivascular Amyloidosis

Synapse Dysfunction

Synaptic Degeneration

Synaptic Loss

Synapse Enlargement

Tau Deposition

Tau Phosphorylation

Tau-Mediated Cytotoxicity
Brain regions affected in $A D$

Amygdala

Anterior Thalamic Nucleus

Basal Nucleus of Meynert

CA1 region

CA2 region

CA3 region

Cholinergic Neuron

Diagonal Band of Broca

Entorhinal Cortex

Frontal Lobe

Hippocampus

Inferior Temporal Gyrus

Left Thalamus

Locus Coeruleus

Medial Temporal Cortex

Parahippocampal Gyrus

Parietal Lobe

Prefrontal Cortex

Septal Nucleus

Subiculum

Substantia Innominata

Superior Temporal Gyrus

Synapse

Temporal Isocortex

Temporal Lobe

Thalamus 


\section{Table 2 Data sources used for the generation of the Intelligence Network}

\begin{tabular}{l} 
Databases \\
\hline Alzheimer Disease \& Frontotemporal Dementia Mutation Database \\
(http://www.molgen.ua.ac.be/ADMutations/Default.cfm)
\end{tabular}

Diseases Database (www.diseasesdatabase.com)

Gene Ontology (www.geneontology.org)

Genetic Association Database (http://geneticassociationdb.nih.gov)

Gensat Brain Atlas (www.gensat.org)

KEGG (www.genome.jp/kegg/pathway.html)

NCBI Gene Expression Omnibus (www.ncbi.nlm.nih.gov/geo)

OMIM (http://www.omim.org/)

Telemakus knowledgebase (http://www.telemakus.net/AD/)

\section{Textual Data}

PubMed (http://www.ncbi.nlm.nih.gov/pubmed/)

\section{Description}

The Alzheimer Disease \& Frontotemporal Dementia Mutation Database (AD\&FTDMDB) aims at collecting all known mutations and non-pathogenic coding variations in the genes related to Alzheimer disease (AD) and frontotemporal dementia (FTD). All data were exported and loaded into Sofia, to create gene-disease assertions.

The Diseases database is a cross-referenced medical dictionary of diseases, medications, symptoms, signs and investigations, which was loaded into Sofia and provided assertions linking Alzheimers disease to symptoms and signs, histopathological abnormalities, risk factors etc.

The Gene Ontology project provides an ontology of defined terms representing gene product properties. The ontology covers three domains for the gene products: cellular component, molecular function, \& biological process. All of GO was processed and loaded into Sofia, and the relevant assertions were then exported into the IN.

The Genetic Association Database is an archive of human genetic association studies of complex diseases and disorders. All the data linking genes to diseases were processed and downloaded into Sofia, and the relevant assertions were then exported into the IN.

GENSAT is a gene expression atlas of the developing and adult central nervous system of the mouse. After AD-related brain areas were identified from literature reviews, the relevant genes were exported from GENSAT, and assertions linking gene to anatomical area created and loaded into Sofia.

KEGG (Kyoto Encyclopedia of Genes and Genomes) is a bioinformatics resource for linking genomes to life and the environment. Pathways relevant to AD were reviewed, and relevant protein-pathway assertions were generated using Sofia.

Gene Expression Omnibus (GEO) is a database repository of high throughput gene expression data and hybridization arrays, chips, microarrays. GEO was searched for AD-relevant expression data, which were downloaded from the NCBI site and loaded into Sofia.

Online Mendelian Inheritance in Man (OMIM) is a database that catalogues all the known diseases with a genetic component, and if possible, links them to the relevant genes in the human genome and provides references for further research and tools for genomic analysis of a catalogued gene. All of OMIM Genemap was exported and loaded into Sofia; relevant AD records were used to create gene-disease assertions.

Telemakus Biomarkers in Alzheimer's Disease \& Mild Cognitive Impairment Knowledgebase contains information from $\mathrm{AD}$ and $\mathrm{MCl}$ biomarker studies. All of the Knowledgebase was exported and loaded into Sofia as protein-disease assertions.

\section{Description}

PubMed is a service of the U.S. National Library of Medicine that includes over 18 million citations from MEDLINE and other life science journals for biomedical articles back to the 1950s. PubMed contains a rich set of biomedical literature abstracts relevant to many areas. AD-relevant vocabularies within Sofia were used to build a "corpus" of AD-relevant abstracts, which were then used in assertion-generation processes to create disease-protein and disease-process links.

Various full text reviews from journals, and reports from AD websites (Alzheimer Research Forum; http://www.alzforum.org/ and Essential Science Indicators; http://www.esi-topics.com/alzheimer/) were downloaded, and text versions were loaded into Sofia for assertion generation using key AD-related vocabularies. subject-verb-object triplets. All proto-assertions were manually checked by trained curators, to ensure that the each component of the triplets had matched appropriately and the assertion was represented accurately in the underlying reference. Inappropriate assertions were discarded. Each curator was able to validate approximately 500 assertions per day. An accuracy level of greater than
97\% was confirmed by random sampling and quality control testing (following an ISO 2859 sampling scheme). For structured sources, generally the whole data source was downloaded and parsed into Sofia with the appropriate relationships, and accuracy levels were checked within an appropriate sample set. Overall, the designbuild-curate-QC process for the IN took a total of three 
months. The resulting IN consisted of over 50,000 assertions, linking more than 200 different pathological observation concepts, over 6,500 protein concepts, and over 35 anatomical/tissue concepts.

\section{Derivation of candidates from intelligence network}

The use of the resulting IN, which essentially represented a semantically-consistent layer over previously disparate information sources, provided an opportunity to apply simple filtering techniques that in this case led to the derivation of a set of proteins fitting the criteria for a candidate biomarker for AD. According the workflow in Figure 2, the IN was first searched for proteins expressed in brain regions of relevance to $\mathrm{AD}$. This set of proteins was then filtered further, retaining only those with assertional evidence for upregulation in AD. Of those proteins, only those that report assertions highlighting an involvement in the development of the pathological hallmarks of AD were retained. The proteins identified as potential biomarkers by this in silico approach were then subjected to an assessment of novelty to see whether relevant publications that discussed the possible use of the biomarkers for AD existed.

\section{In vitro assessment of candidate biomarkers \\ Samples}

In order to validate candidate proteins identified in the in silico study we utilized samples from the multisite European AddNeuroMed cohort [11]. A total of 300

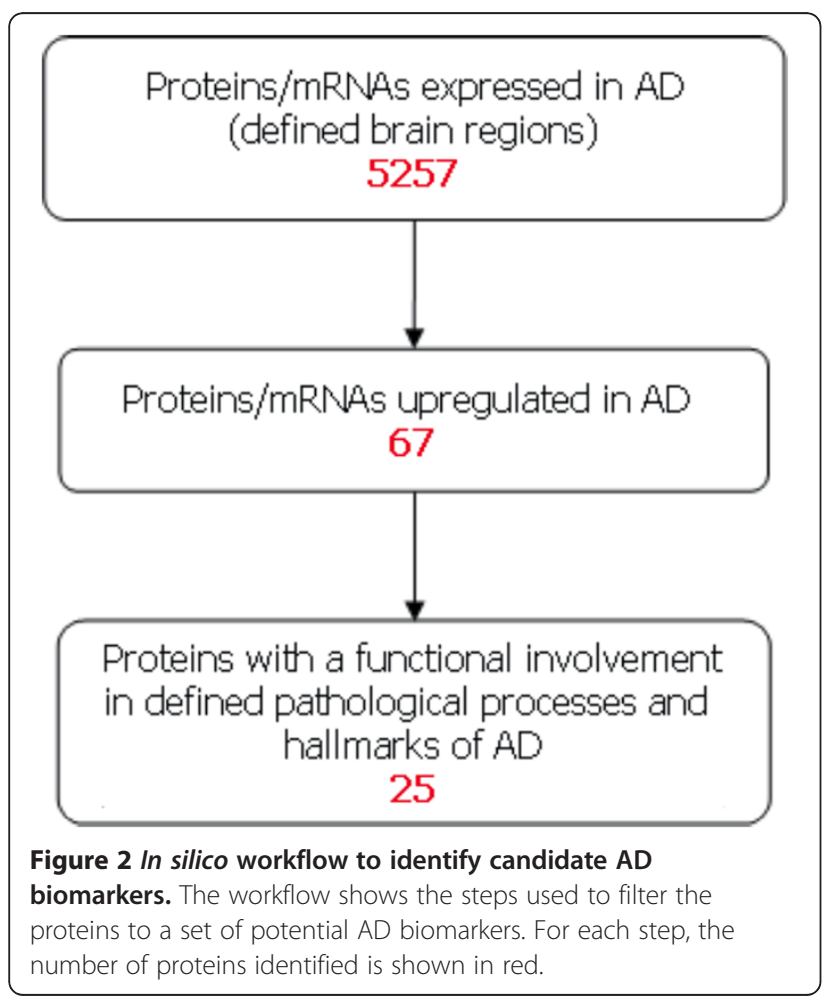

samples with imaging and clinical data available including 100 each of $\mathrm{AD}$ cases, normal elderly controls, and MCI cases and collected from 6 locations across Europe (Kuopio, Finland; London, United Kingdom; Perugia, Italy; Toulouse, France; Lodz, Poland; and Thessaloniki, Greece) were used with all biochemistry assessments being blind to disease status. The AddNeuroMed study including diagnostic process, data and sample collection and curation protocols and imaging processes are described elsewhere [11-13].

\section{Imaging}

The imaging protocols and analysis of MRI within AddNeuroMed is described elsewhere as noted above. In brief, MR images are subjected to automated analysis following parcellation of the brain in a pipeline that results in over 70 regional cortical volumes and thickness of grey matter variables. Correlational and other analysis of these variables have shown similar diagnostic and predictive qualities as in other, comparable studies such as ADNI $[14,15]$. Variables that contribute most to diagnostic accuracy include, unsurprisingly those reflecting atrophy in areas of brain known to be most affected by $\mathrm{AD}$ including entorhinal cortex and hippocampus.

\section{Protein quantization in plasma}

We performed semi-quantitative immmunoblotting of diluted (1:50) plasma for two candidate proteins as a validity test process for the in silico study. The immunoblotting protocol has been previously described [7] but in brief involved separation by SDS-PAGE, transfer to nitrocellulose, incubation with primary antibody and identification with secondary antibody. Antibodies used in this study included those for ChAt, mouse monoclonal anti-human ChAt antibody (Chemicon Clone 1. B3.9B3) and for PLAUR mouse monoclonal anti-human PLAUR antibody (R\&D, CD87, cat.no. MAB807).

Blots were then scanned with Odyssey Infrared Imaging (Li-Cor Bioscience) scanner and analyzed using Odyssey Infrared Imaging System Version 1.2. The Integrated Intensity for each band of the relevant molecular weight was determined and background subtracted.

\section{Statistical analysis}

The Integrated Intensity value for both ChAt and PLAUR, corrected for protein loading was analyzed using SPSS (v19). Following tests to assess the normality of data distribution (Kolmogorov-Smirnov and ShapiroWilk), we first used ANOVA to determine the relationship between protein level and case status and then performed bivariate correlation (Spearman rank correlation) between protein values and brain atrophy measured using MRI. 


\section{Results}

In silico discovery of candidate biomarkers

In order to identify potential peripheral AD biomarkers we first performed a consensus building exercise which suggested that an AD biomarker might have the characteristics of being a protein expressed in areas of the brain known to be affected by $\mathrm{AD}$ and to be associated with a pathological process relevant to $\mathrm{AD}$ and to be differentially expressed in $\mathrm{AD}$. We then utilized text mining and linguistics analysis to construct the 'AD Biomarker Intelligence Network' from meta-assertional data derived from all major publically available biological datasets. The workflow described in Figure 2 revealed the following:

- 5,257 proteins and/or mRNAs expressed in $\mathrm{AD}$ tissue

- of these, 67 reported evidence for upregulation in $\mathrm{AD}$
- of these, 25 have known associations with a pathological process in $\mathrm{AD}$

These candidate biomarker proteins are listed in Table 3.

\section{In vitro assessment of candidate biomarkers}

In order to validate the in silico approach to potential biomarker discovery, we chose two proteins not previously suggested as peripheral biomarkers for AD PLAUR and ChAt - and determined the levels of these proteins in plasma from 240 subjects from the AddNeuroMed cohort, a European AD biomarkers study, using semi-quantitative immunoblotting. Only two proteins not previously associated with $\mathrm{AD}$ were tested and these two were chosen partly as one (PLAUR) is a known plasma protein altered in other disease states and one is present in plasma but had not previously

Table 3 Final set of derived proteins representing candidate biomarker of AD

\begin{tabular}{|c|c|c|}
\hline & $\begin{array}{l}\text { STANDARD } \\
\text { PROTEIN SYMBOL }\end{array}$ & COMMON ALIASES \\
\hline Alpha 1-antichymotrypsin & SERPINA3 & serpin peptidase inhibitor clade $A, A A C T, A C T$ \\
\hline $\begin{array}{l}\text { Amyloid beta (A4) precursor protein (protease nexin-II, } \\
\text { Alzheimer disease amyloid protein) }\end{array}$ & APP & AD1 (Alzheimer disease), ABETA (amyloid beta A4 protein) \\
\hline Apolipoprotein D & APOD & \\
\hline Apolipoprotein E & apoE & AD2 (Alzheimer disease 2) \\
\hline B-cell leukemia/lymphoma 2 & $\mathrm{BCL} 2$ & \\
\hline Beta-site APP-Cleaving Enzyme 1 & BACE1 & \\
\hline Butyrylcholinesterase & $\mathrm{BCHE}$ & \\
\hline C-reactive protein, pentraxin-related & CRP & \\
\hline Choline Acetyltransferase & CHAT & CHOACTase \\
\hline Clusterin & CLU & APOJ (Apolipoprotein J) \\
\hline Complement component 1, q subcomponent, beta polypeptide & $\mathrm{C} 1 \mathrm{QB}$ & \\
\hline Estrogen Receptor 1 (alpha) & ESR1 & \\
\hline Glial fibrillary acidic protein & GFAP & \\
\hline Heat shock 70kD protein 5 (glucose-regulated protein) & HSPA5 & \\
\hline Interleukin 1 beta & IL1B & \\
\hline Interleukin 6 & IL6 & IFNB2 (Interferon beta-2) \\
\hline Matrix Metallopeptidase 9 & MMP9 & CLG4B (92 kDa gelatinase, 92 kDa type IV collagenase) \\
\hline Nerve Growth Factor & NGF & NGFB \\
\hline Nitric Oxide Synthase 2A & NOS2 & NOS2A, INOS (Inducible NOS), HEP-NOS (Hepatocyte NOS) \\
\hline PRKC, apoptosis, WT1, regulator & PAWR & PAR4 (Prostate apoptosis response 4 protein) \\
\hline Prostaglandin-Endoperoxide Synthase 2 & PTGS2 & PGHS2, COX2 (Cyclooxygenase 2b) \\
\hline Transforming Growth Factor, Beta 1 & TGFB1 & \\
\hline Transthyretin & $T T R$ & \\
\hline Tumor Necrosis Factor (TNF superfamily, member 2) & TNF & TNFA, TNF-alfa \\
\hline Urokinase Plasminogen Activator Receptor & PLAUR & UPAR \\
\hline
\end{tabular}


been investigated as a biomarker for any condition to our knowledge and partly for serendipitous reasons antibodies suitable for assay generation were readily available. We aimed first to compare protein levels in research participants with $\mathrm{AD}$, with $\mathrm{MCI}$ and normal elderly controls. In addition, as $\mathrm{AD}$ has a long prodromal phase and as a consequence many apparently normal elderly people have substantial but occult AD pathology, we also correlated protein levels with cerebral atrophy measured using automated analysis of structural MRI (191 subjects with imaging data) as a quantitative marker of pathological load independent of clinical disease status.

Table 4 shows the diagnostic category, age and gender characteristics of the subjects analyzed together with the results of PLAUR and ChAt analysis. We found PLAUR to be highly significantly different across categories with lowest levels in $\mathrm{AD}$, highest in controls and an intermediate level in MCI (ANOVA, $\mathrm{p}<0.001$ ). There was no difference in ChAT comparing the three diagnostic categories. To estimate degree of pathology regardless of diagnostic category we used whole brain volume as a measure of atrophy, derived using automated analysis of structural MRI as previously reported [12,14,15]. For PLAUR we found a very highly significant inverse correlation between whole brain volume and protein levels in plasma. Interestingly the AD group showed a similar direction of effect although this was not significant. For ChAt we also found a highly significant, but in this case positive, correlation between whole brain volume and plasma protein levels in control subjects with again the AD group showing a trend towards the same direction of correlation. In neither case was there any correlation in the MCI group.

\section{Discussion}

The discovery of biomarkers for $\mathrm{AD}$ is an increasingly important task - both for early diagnosis and for use in experimental medicine. However, it is a task complicated by at least three major intrinsic difficulties. First the complexity of AD pathology means that identification of candidate markers, beyond the low hanging fruit of $A \beta$ and tau, is problematical. Second, collection of the optimal peripheral fluid for biomarker identification, CSF, is relatively invasive and unsuitable for repeated measures in elderly people. Third, AD has a prolonged prodrome when apparently normal elderly people harbor considerable pathological load meaning that the conventional case-control design is confounded by pathology in clinically unaffected subjects. Previously we and others have attempted to mitigate the third of these complications by using a design of biomarker discovery where the independent or outcome variable is not clinical diagnosis but an endophenotype of disease such as structural MRI evidence of atrophy [7] or PET evidence of A $\beta$ load [6]. The second of the limitations in biomarkers for $\mathrm{AD}$ the availability of CSF - has prompted many groups to seek markers in other fluids such as plasma. The first of the limitations, the identification of candidates, has been previously attempted by two broad categories of studies; either using candidates based on the researcher's own understanding of disease or using a data-driven, most often proteomic, approach. Here we combine the use of endophenotypes to complement diagnostic category as an outcome measure, with the use of plasma as a biomarker tissue, with an entirely novel approach to the identification of candidates. This innovation makes use of linguistic and textual analysis to interrogate the entire biomedical knowledge base in the form of all the major publicly available databases to identify candidate markers using a consensus driven set of primary assertions. We accessed the various data sources in 2006, and clearly in a fast moving field data will have changed considerably in the intervening years. Indeed some of the proteins identified in 2006 (such as Transthyretin and Clusterin) had not at that time been considered as biomarkers. However, when the in vitro analysis was carried out, data had been provided from our own proteomics studies that these proteins were in fact putative biomarkers. Thus this time lag has inadvertently provided further substantiation of the proof of concept of the in silico approach that we discuss here.

The textual analysis of publicly available data sources suggested a total of 25 potential candidate biomarkers.

Table 4 Subject characteristics and PLAUR and ChAt analysis in validation study

\begin{tabular}{|c|c|c|c|c|c|c|c|c|c|c|}
\hline & \multirow[b]{2}{*}{$\mathrm{N}$} & \multirow[b]{2}{*}{ Gender (\% F) } & \multirow[b]{2}{*}{ Age mean (SD) } & \multirow[b]{2}{*}{ MMSE* mean (SD) } & \multirow[b]{2}{*}{ PLAUR mean (SD) ** } & \multicolumn{2}{|c|}{$\begin{array}{l}\text { PLAUR } \\
\text { correlation with } \\
\text { whole brain } \\
\text { volume }\end{array}$} & \multirow[b]{2}{*}{ ChAt mean (SD) ** } & \multicolumn{2}{|c|}{$\begin{array}{c}\text { ChAt } \\
\text { correlation } \\
\text { with whole } \\
\text { brain volume }\end{array}$} \\
\hline & & & & & & $\mathbf{R}$ & $p$ & & $\mathbf{R}$ & $p$ \\
\hline Controls & 82 & 58 & $72.8(7.0)$ & $29(1.1)$ & $1.63(0.9)$ & -0.35 & $<0.005$ & $0.78(0.3)$ & 0.34 & $<0.01$ \\
\hline $\mathrm{MCl}$ & 80 & 57 & $74.7(6.2)$ & $27(2.2)$ & $0.98(0.7)$ & 0.14 & NS & $0.75(0.3)$ & 0.01 & NS \\
\hline$A D$ & 78 & 69 & $76.2(6.4)$ & $21(4.5)$ & $0.85(0.3)$ & -0.2 & NS & $0.71(0.3)$ & 0.23 & 0.06 \\
\hline
\end{tabular}

*MMSE; Mini mental state examination - a cognitive scale (maximum score $=30$ ) used to screen for and assess degree of severity of dementia. A score of less than 24 indicates possible dementia. A score of $10-20$ suggests moderate dementia and a score above 20 , mild dementia. ** arbitrary units. 
Some of these have previously been identified as potential biomarkers in plasma. For example, using MRI measures of atrophy as an outcome endophenotype we identified and confirmed plasma Clusterin [7] and Transthyretin [16] as measure of severity of disease and using PET measures of amyloid identified apoE protein as the primary correlate in plasma [6]. All these studies used gel based proteomics as the discovery tool and the fact that textual analysis identifies the same proteins before these proteomic studies were performed is a strong indicator of the power of the method. Other promising candidates suggested by textual analysis, and where there is published data suggesting that these proteins are altered either in blood or CSF, include CRP [17-19], Complement factor 1 [20,21], butyrylcholinesterase [22] and BACE1 $[23,24]$. In all but the case of butyrylcholinesterase, this biomarker data was published after the IN lockdown and hence these biomarker utility data are independent of the IN and act as independent proof of concept.

As the IN identified as potential protein biomarkers proteins previously identified in proteomic studies without this data entering into this particular network we were encouraged to attempt further validation in plasma. We chose two proteins - neither previously identified as potential plasma biomarkers to our knowledge - and measured these in over 200 subjects most of whom had as part of the European AddNeuroMed project, automated analysis of structural MRI data available. One of these proteins - PLAUR - was significantly decreased in AD relative to controls with MCI being at an intermediate level. Both PLAUR and ChAT showed a correlation, inverse in the case of PLAUR, with imaging evidence of atrophy in control cases and both showed a smaller and non-significant, but in the same direction, correlation in AD cases. We used semi-quantitative immunoblotting as a screening method as in previous studies as this approach, in contrast to ELISA for example, yields information on degradation products and post translational modifications. In fact the data on these two chosen proteins suggested whole protein correlation with disease state suggesting future biomarker replication and qualification studies, beyond the intention of the present investigation, might progress rapidly to fully quantitative methods.

Urokinase plasminogen activator receptor (PLAUR) is a protein involved in many biological functions including cell signaling $[25,26]$. By binding urokinase plasminogen activator (uPA), with which it forms an active complex (uPA-PLAUR), it catalyzes the transformation of zymogen plasminogen into the active protein plasmin, a serine protease which degrades fibrin. The receptor is also involved in cell signaling and in chemotaxis, and controls cell adhesion. Increased levels of PLAUR have been previously reported in inflammatory disorders [27] and has been implicated in chemotaxis leading to microglial accumulation in the core of amyloid plaques in brain in transgenic rodent models of $A D$. $A \beta$ induces PLAUR [28] and PLAUR is increased in microglia cells of human $\mathrm{AD}$ brains and in brains treated with amyloid $\beta$ peptide $[29,30]$. The inverse relationship we observe between soluble PLAUR and AD and brain atrophy is noteworthy and might suggest an inverse relationship either between soluble and, functional, membrane bound PLAUR or between central and peripheral PLAUR more generally. An inverse relationship between central amyloid load and peripheral, CSF, amyloid has been previously and extensively noted. The other novel protein association with pathology we identify, ChAt, is a key component of the cholinergic pathway which is severely affected in $\mathrm{AD}$ and is the target for the first symptomatic therapies for $\mathrm{AD}$. We observe a relationship between cerebral atrophy and ChAT protein and this may reflect the loss of cholinergic neurons known to occur early in disease process.

In summary we show here that the extraction of data from huge volumes of biological datasets including text based information is possible and that the creation of hypothesis or assertion-driven analysis yields potential biomarkers. As some of these markers have been independently generated using proteomics, and, as here we show at least partial validation of the two markers tested, this finding offers strong support to a text mining approach to biomarker discovery using the ever increasing publically available datasets.

\section{Competing interests}

KCL has protected intellectual property related to biomarkers for AD but unrelated to the work described in this paper, BioWisdom has intellectual property interests in information technology related to the work described in this paper.

\section{Authors' contributions}

$N D$, JR performed the data-mining and in silico analysis. IG, JR-C performed the biomarker assay studies. IG, SL drafted the manuscript. IG, ND, JR, JR-C, $H S, A S$, JB, SL edited the manuscript. ND, HS, AS, JB, SL participated in study design. HS, IK, MT, BV, CS, PM, L-OW, AS, SL coordinated the clinical study collection of samples and clinical data. All authors read and approved the final manuscript

\section{Acknowledgements}

We are grateful for funding to the AddNeuroMed/InnoMed, (Innovative Medicines in Europe) an Integrated Project funded by the European Union of the Sixth Framework program priority FP6-2004-LIFESCIHEALTH-5, Life Sciences, Genomics and Biotechnology for Health and to the NIHR Biomedical Research Centre for Mental Health at the South London and Maudsley NHS Foundation Trust.

\section{Author details}

'King's College London, Institute of Psychiatry, De Crespigny Park, London SE5 8AF, UK. ${ }^{2}$ BioWisdom Ltd, Cambridge, UK (now Instem Scientific). ${ }^{3}$ University of Eastern Finland and University Hospital of Kuopio, Kuopio, Finland. ${ }^{4}$ Medical University of Lodz, Lodz, Poland. ${ }^{5}$ 3rd Department of Neurology, "G. Papanicolaou" Hospital, Aristotle University of Thessaloniki, Thessaloniki, Greece. ' UMR INSERM 1027, Gerontopole, CHU Toulouse, 
University of Toulouse, Toulouse, France. ${ }^{7}$ Department of Clinical Science, Intervention and Technology, Karolinska Institutet, Stockholm, Sweden. ${ }^{8}$ Institute of Gerontology and Geriatrics, University of Perugia, Perugia, Italy. ${ }^{9}$ Department of Neurobiology, Care Sciences and Society, Karolinska Institutet, Stockholm, Sweden. ${ }^{10}$ Currently at Somaxa Ltd and Abcodia Ltd, London, UK.

Received: 28 March 2012 Accepted: 14 September 2012

Published: 31 October 2012

\section{References}

1. Lovestone S, Guntert A, Hye A, Lynham S, Thambisetty M, Ward M: Proteomics of Alzheimer's disease: understanding mechanisms and seeking biomarkers. Expert Rev Proteomics 2007, 4(2):227-238.

2. Hampel H, Wilcock G, Andrieu S, Aisen P, Blennow K, Broich K, Carrillo M, Fox NC, Frisoni GB, Isaac M, et al: Biomarkers for Alzheimer's disease therapeutic trials. Prog Neurobiol 2011, 95(4):579-593.

3. Blennow K, Hampel H, Weiner M, Zetterberg H: Cerebrospinal fluid and plasma biomarkers in Alzheimer disease. Nat Rev Neurol 2010, 6(3):131-144.

4. Verwey NA, Van Der Flier WM, Blennow K, Clark C, Sokolow S, De Deyn PP, Galasko D, Hampel H, Hartmann T, Kapaki E, et al: A worldwide multicentre comparison of assays for cerebrospinal fluid biomarkers in Alzheimer's disease. AnnClinBiochem 2009, 46(Pt 3):235-240.

5. Thambisetty M, Lovestone S: Blood-based biomarkers of Alzheimer's disease: challenging but feasible. BiomarkMed 2010, 4(1):65-79.

6. Thambisetty M, Tripaldi R, Riddoch-Contreras J, Hye A, An Y, Campbell J, Sojkova J, Kinsey A, Lynham S, Zhou Y, et al: Proteome-based plasma markers of brain amyloid-beta deposition in non-demented older individuals. J Alzheimers Dis 2010, 22(4):1099-1109.

7. Thambisetty M, Simmons A, Velayudhan L, Hye A, Campbell J, Zhang Y, Wahlund LO, Westman E, Kinsey A, Guntert A, et al: Association of plasma clusterin concentration with severity, pathology, and progression in Alzheimer disease. ArchGenPsychiatry 2010, 67(7):739-748.

8. Klein J, Jupp S, Moulos P, Fernandez M, Buffin-Meyer B, Casemayou A, Chaaya R, Charonis A, Bascands JL, Stevens R, et al: The KUPKB: a novel Web application to access multiomics data on kidney disease. FASEB $J$ 2012, 26(5):2145-2153.

9. Reorganizing the protein space at the Universal Protein Resource (UniProt): Nucleic Acids Res 2012, 40(Database issue):D71-75.

10. Fourches D, Barnes JC, Day NC, Bradley P, Reed JZ, Tropsha A: Cheminformatics analysis of assertions mined from literature that describe drug-induced liver injury in different species. Chem Res Toxicol 2010, 23(1):171-183.

11. Lovestone $S$, Francis $P$, Kloszewska I, Mecocci P, Simmons A, Soininen $H$, Spenger C, Tsolaki M, Vellas B, Wahlund LO, et al: AddNeuroMed-the European collaboration for the discovery of novel biomarkers for Alzheimer's disease. AnnNYAcadSci 2009, 1180:36-46.

12. Simmons A, Westman E, Muehlboeck S, Mecocci P, Vellas B, Tsolaki M, Kloszewska I, Wahlund LO, Soininen H, Lovestone S, et al: The AddNeuroMed framework for multi-centre MRI assessment of Alzheimer's disease: experience from the first 24 months. Int I Geriatr Psychiatry 2011, 26(1):75-82.

13. Simmons A, Westman E, Muehlboeck S, Mecocci P, Vellas B, Tsolaki M, Kloszewska I, Wahlund LO, Soininen H, Lovestone S, et al: MRI measures of Alzheimer's disease and the AddNeuroMed study. AnnNYAcadSci 2009, 1180:47-55.

14. Westman E, Simmons A, Muehlboeck JS, Mecocci P, Vellas B, Tsolaki M, Kloszewska I, Soininen $\mathrm{H}$, Weiner MW, Lovestone $\mathrm{S}$, et al: AddNeuroMed and ADNI: Similar patterns of Alzheimer's atrophy and automated MRI classification accuracy in Europe and North America. Neuroimage 2011, 58(3):818-828

15. Westman E, Simmons A, Zhang Y, Muehlboeck JS, Tunnard C, Liu Y, Collins $L$, Evans A, Mecocci P, Vellas B, et al: Multivariate analysis of MRI data for Alzheimer's disease, mild cognitive impairment and healthy controls. Neuroimage 2011, 54(2):1178-1187.

16. Velayudhan L, Killick R, Hye A, Kinsey A, Guentert A, Lynham S, Ward M, Leung $R$, Lourdusamy $A$, To AW, et al: Plasma transthyretin as a candidate marker for Alzheimer's disease. J Alzheimers Dis 2012, 28(2):369-375.

17. Mancinella A, Mancinella M, Carpinteri G, Bellomo A, Fossati C, Gianturco V, Iori A, Ettorre E, Troisi G, Marigliano V: Is there a relationship between high
C-reactive protein (CRP) levels and dementia? Arch Gerontol Geriatr 2009, 49(Suppl 1):185-194

18. Kravitz BA, Corrada MM, Kawas CH: Elevated C-reactive protein levels are associated with prevalent dementia in the oldest-old. Alzheimers Dement 2009, 5(4):318-323.

19. Davis G, Baboolal N, Nayak S, McRae A: Sialic acid, homocysteine and CRP: potential markers for dementia. Neurosci Lett 2009, 465(3):282-284.

20. Schjeide BM, Schnack C, Lambert JC, Lill CM, Kirchheiner J, Tumani H, Otto $M$, Tanzi RE, Lehrach $H$, Amouyel $P$, et al: The role of clusterin, complement receptor 1 , and phosphatidylinositol binding clathrin assembly protein in Alzheimer disease risk and cerebrospinal fluid biomarker levels. Arch Gen Psychiatry 2011, 68(2):207-213.

21. Lambert JC, Heath S, Even G, Campion D, Sleegers K, Hiltunen M, Combarros O, Zelenika D, Bullido MJ, Tavernier B, et al: Genome-wide association study identifies variants at CLU and CR1 associated with Alzheimer's disease. Nat Genet 2009, 41(10):1094-1099.

22. Parnetti L, Amici S, Lanari A, Romani C, Antognelli C, Andreasen N, Minthon L, Davidsson P, Pottel H, Blennow K, et al: Cerebrospinal fluid levels of biomarkers and activity of acetylcholinesterase (AChE) and butyrylcholinesterase in $A D$ patients before and after treatment with different AChE inhibitors. NeurolSci 2002, 23(Suppl 2):S95-S96.

23. Ewers M, Zhong Z, Burger K, Wallin A, Blennow K, Teipel SJ, Shen Y, Hampel H: Increased CSF-BACE 1 activity is associated with ApoE-epsilon 4 genotype in subjects with mild cognitive impairment and Alzheimer's disease. Brain 2008, 131(Pt 5):1252-1258.

24. Kassner SS, Bonaterra GA, Kaiser E, Hildebrandt W, Metz J, Schroder J, Kinscherf R: Novel systemic markers for patients with Alzheimer disease? - a pilot study. Curr Alzheimer Res 2008, 5(4):358-366.

25. Blasi F, Sidenius N: The urokinase receptor: focused cell surface proteolysis, cell adhesion and signaling. FEBS Lett 2010, 584(9):1923-1930

26. Smith HW, Marshall CJ: Regulation of cell signalling by uPAR. Nat Rev Mol Cell Biol 2010, 11(1):23-36.

27. Blasi F, Carmeliet P: UPAR: a versatile signalling orchestrator. Nat Rev Mol Cell Biol 2002, 3(12):932-943.

28. Davis J, Wagner MR, Zhang W, Xu F, Van Nostrand WE: Amyloid betaprotein stimulates the expression of urokinase-type plasminogen activator (UPA) and its receptor (UPAR) in human cerebrovascular smooth muscle cells. J Biol Chem 2003, 278(21):19054-19061.

29. Walker DG, Lue LF, Beach TG: Increased expression of the urokinase plasminogen-activator receptor in amyloid beta peptide-treated human brain microglia and in AD brains. Brain Res 2002, 926(1-2):69-79.

30. Asahina M, Yoshiyama Y, Hattori T: Expression of matrix metalloproteinase- 9 and urinary-type plasminogen activator in Alzheimer's disease brain. Clin Neuropathol 2001, 20(2):60-63.

\section{doi:10.1186/1479-5876-10-217}

Cite this article as: Greco et al: Alzheimer's disease biomarker discovery using in silico literature mining and clinical validation. Journal of Translational Medicine 2012 10:217.

\section{Submit your next manuscript to BioMed Central and take full advantage of:}

- Convenient online submission

- Thorough peer review

- No space constraints or color figure charges

- Immediate publication on acceptance

- Inclusion in PubMed, CAS, Scopus and Google Scholar

- Research which is freely available for redistribution 